\title{
REVIEW ARTICLE The impact of a helminth-modified microbiome on host immunity
}

\author{
Tara P. Brosschot ${ }^{1}$ and Lisa A. Reynolds ${ }^{1}$
}

\begin{abstract}
Intestinal helminths have well-characterized modulatory effects on mammalian immune pathways. Ongoing helminth infection has been associated with both the suppression of allergies and an altered susceptibility to microbial infections. Enteric helminths share a niche with the intestinal microbiota, and the presence of helminths alters the microbiota composition and the metabolic signature of the host. Recent studies have demonstrated that the helminth-modified intestinal microbiome has the capacity to modify host immune responses even in the absence of live helminth infection. This article discusses the mechanisms by which helminths modify the intestinal microbiome of mammals, and reviews the evidence for a helminth-modified microbiome directly influencing host immunity during infectious and inflammatory diseases. Understanding the multifaceted mechanisms that underpin helminth immunomodulation will pave the way for novel therapies to combat infectious and inflammatory diseases.
\end{abstract}

Mucosal Immunology (2018) 11:1039-1046; https://doi.org/10.1038/s41385-018-0008-5

\section{INTRODUCTION}

Helminths are multicellular parasitic worms that remain a global health concern; billions of people are currently infected worldwide. These parasites can mount long-lasting infections, often spanning years, which has been attributed to their ability to manipulate mammalian immune responses and thereby avoid immune-mediated expulsion. ${ }^{1}$

Modulation of mammalian immune responses by helminths not only promotes their own persistence, but can additionally affect host immunity to concurrent infections and responses to bystander antigens. For example, helminth infection has been associated with impaired immunity to coinfection with various microbial parasites, bacteria, and viruses, both in human populations $^{2-6}$ and in mouse infection models. ${ }^{7-15}$ Given that chronic helminth infections are predominantly restricted to regions of poor sanitation where people have a high exposure to microbial pathogens, ${ }^{16}$ understanding how helminth infection modulates host responses to microbial coinfection is critical. In parallel, live helminth infection has been associated with a reduced prevalence of allergic disease in human populations, ${ }^{17}$ and suppression of allergic airway inflammation in mouse disease models. ${ }^{18-21}$ Understanding how helminths modulate host immunity is therefore of global importance, as triggering the immune pathways helminths target has the potential for exploitation in the treatment of allergic conditions, which are a growing burden on the healthcare system in industrialized countries. ${ }^{22}$

Helminth infection typically elicits a type 2 immune response, characterized by the production of IL-4, IL-5, IL-9, and IL-13 from type 2 innate lymphoid cells (ILC2) and T helper 2 (Th2) cells, and the recruitment of eosinophils, mast cells, basophils, and alternatively activated macrophages. ${ }^{23}$ Type 2 effector mechanisms are essential for immune-mediated helminth expulsion, yet are often ineffective, largely due to concurrent regulatory responses induced by helminth infection. For example, helminths release a variety of so-called excretory-secretory (ES) products, which include immunomodulatory proteins, glycoproteins, and miRNAs, ${ }^{24,25}$. Helminth ES products have been reported to modulate the activity of multiple immune cell types, and can directly induce regulatory immune cells, such as Foxp $3^{+}$regulatory $\mathrm{T}$ cells (Tregs). ${ }^{1,26}$ Production of ES material by helminths likely underpins the longevity of helminths in their host, as well as modulating host susceptibility to inflammatory and infectious diseases. $^{27}$

Intestine-dwelling helminths are not alone in the gut. Mammalian intestines harbor a vast array of mutualistic and commensal microbes, collectively termed the microbiota, which includes species of bacteria, viruses, and fungi. Helminth infection not only affects the composition of microbiota species, but as a result, infection alters the array of products derived from the microbiota. In this article, we define the 'intestinal microbiome' as the microbiota species themselves, their genes and products, as well as the products in their surrounding intestinal environment. Several recent studies have investigated how shifts in the intestinal microbiome during helminth infection contribute to the immunomodulatory effects of helminth infection. Below, we discuss the mechanisms by which helminths alter the microbiome, and review the evidence for a helminth-modulated microbiome directly affecting host immunity during infectious or inflammatory disease conditions.

\section{HELMINTHS MODIFY THE BACTERIAL INTESTINAL MICROBIOTA}

The bacterial microbiota provide several functions that are vital for the health of mammals. Their presence is required for the development of normal gut architecture, ${ }^{28}$ for stimulating immune development and for maintaining normal immune homeostasis. ${ }^{29}$ Compositional differences within the microbiota

\footnotetext{
${ }^{1}$ Department of Biochemistry and Microbiology, University of Victoria, Victoria, BC V8P 5C2, Canada
}

Correspondence: Lisa A. Reynolds (lisareynolds@uvic.ca)

Received: 30 September 2017 Revised: 19 December 2017 Accepted: 22 December 2017

Published online: 16 February 2018 


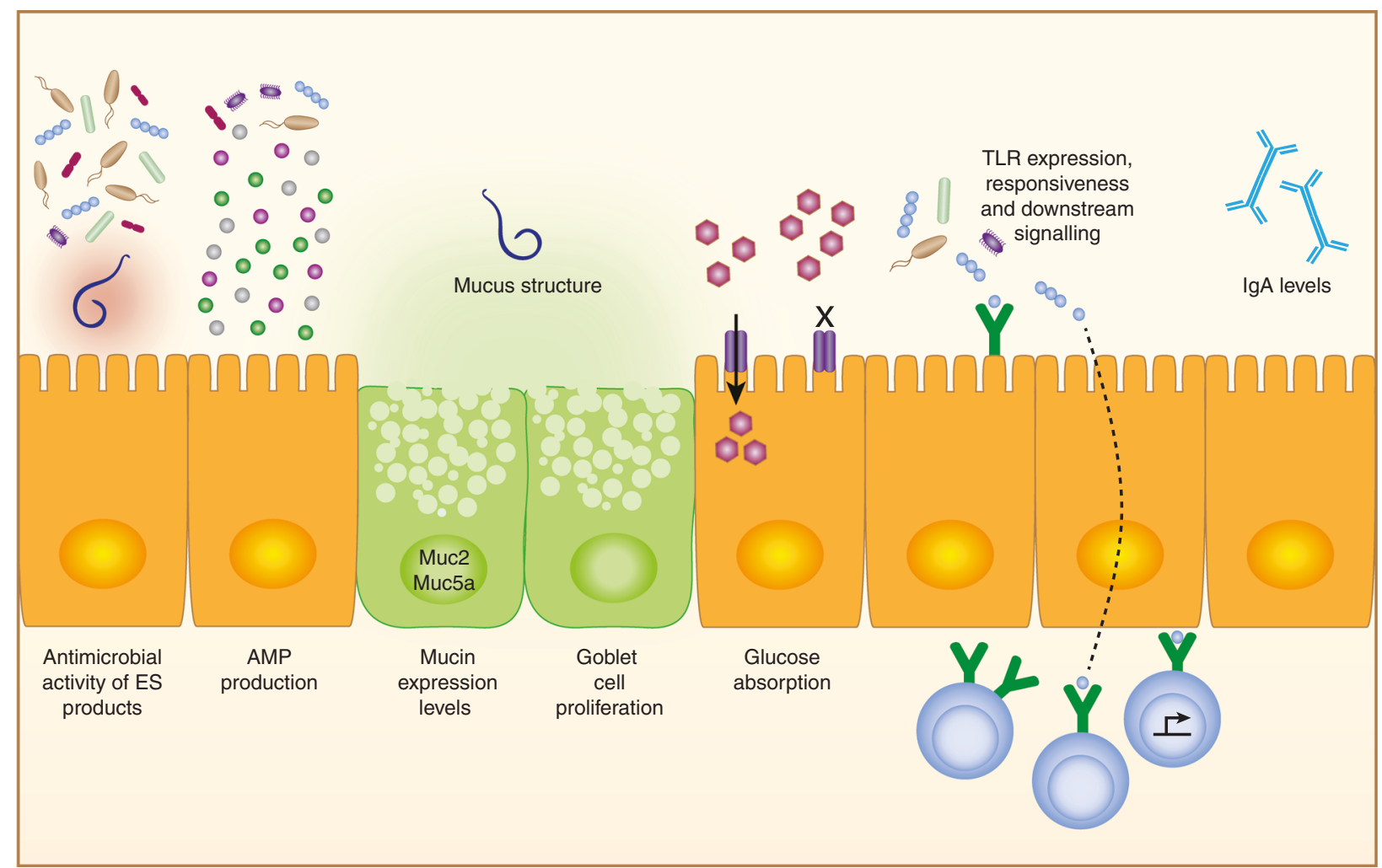

Fig. 1 Parameters modified during helminth infection which may contribute to intestinal microbiota modification. Helminths may alter the microbiota composition directly, through the antimicrobial activity of their ES products, ${ }^{55-57}$ as well as indirectly, through altering host production of anti-microbial peptides (AMPs). ${ }^{15,48,62-64}$ Intestinal mucus can provide a niche and energy source for certain microbiota species, and the presence of helminths can affect host mucus in several ways: through affecting mucus-producing goblet cell proliferation, ${ }^{100}$ and through altering mucin composition, decoration, and degradation. ${ }^{65,101,102}$ In addition, the availability of intestinal nutrients may be affected during helminth colonization, as compromised epithelial glucose absorption has been reported in the presence of helminths. ${ }^{58}$ Furthermore, helminths and helminth-derived products can regulate host recognition of microbes through influencing host pattern-recognition receptor signaling, such as through altering the expression patterns of TLRs, ${ }^{2,103-105}$ and through modulating the responsiveness of immune cells to TLR ligands. ${ }^{106}$ Helminths may also affect microbiota composition through interference with the production of microbe-specific lgA in the intestine. ${ }^{2}$ ES excretory-secretory, AMP anti-microbial peptide, TLR toll-like receptor, Ig immunoglobulin

have also been shown to fine-tune steady-state immune cell differentiation, for example, specific microbiota species have been associated with the differentiation of mucosal Th17 cells $^{30-32}$ and Tregs. ${ }^{32-35}$ Compositional differences within the bacterial microbiota of individuals have been associated with differing levels of susceptibility to immune-driven diseases, such as allergies and inflammatory bowel disease (IBD). ${ }^{36,37}$ The bacterial microbiota also provide protection from colonization with pathogenic microbes, either directly, by producing toxins or competing for nutrients and space, or indirectly, by promoting intestinal barrier function. ${ }^{38,39}$ Further, members of the bacterial microbiota are able to metabolize dietary components that mammalian enzymes are incapable of digesting. ${ }^{40}$

Helminth infection disrupts the composition of the microbiota within their mammalian hosts, as has been reported in several different animal-helminth systems (recently reviewed in ref. ${ }^{41}$ ). Species within the bacterial family Lactobacillaceae, which are capable of inducing host regulatory responses, ${ }^{42}$ have been widely reported to increase in abundance during helminth infection, irrespective of helminth or host species. ${ }^{43-50}$ Further studies have demonstrated that the presence of Lactobacilli species can promote the persistence of helminth infection, providing evidence for a mutualistic relationship between helminths and Lactobacilli species. ${ }^{43,45,51}$ Aside from the commonly described upregulation of Lactobacillaceae family members, reported helminth-elicited microbiota taxonomical changes vary greatly between studies. Such variation likely arises from a number of factors, not least the species and infection intensity of the colonizing helminth, and their host species. The life cycle, size, and niche of helminths within their mammalian host vary drastically between helminth species, and will have a profound impact on their interaction with the microbiota population of the host. Further, the site at which the microbiota is sampled (for example fecal or intestinal sections), and the sampling time-point relative to helminth exposure will affect the recorded impact on microbiota composition, since helminth-elicited taxonomical shifts vary over the course of infection in mice. ${ }^{43,47,52}$ In addition, baseline differences in microbiota composition between individuals, that could result from a multitude of factors including host diet, $^{53,54}$ will contribute to the impact of helminth infection on microbiota populations.

Enteric helminths may affect the structure of intestinal microbial communities through multiple parallel routes (Fig. 1). Helminths may modify microbiota composition through the direct antimicrobial activity of their ES products, which has been predicted or demonstrated for components of the ES material of several helminth species. ${ }^{55-57}$ In addition, the presence of helminths can alter both nutrient and niche availability in the intestine. For example, epithelial glucose absorption has been reported to be impaired during helminth infection, ${ }^{58}$ which likely favor microbiota species that ferment sugars. Intestinal mucus plays a major role in controlling microbiota populations, ${ }^{29}$ and changes in mucus dynamics during helminth infection could also favor the growth of select microbial species. For example, mucus-colonizing 
Mucispirillum species ${ }^{59}$ are increased in abundance in Trichuris muris-infected mice and Trichuris suis-infected pigs, ${ }^{47,52,60}$ and helminth-induced mucus production has been associated with a bloom of Clostridial species. ${ }^{61}$ Finally, helminth infection may modify both innate and adaptive host immune responses that can promote clearance of intestinal microbes.

Several parameters that likely have downstream effects on microbiota composition are dependent on the type 2 immune response induced during helminth infection. For example, changes in host expression of antimicrobial peptides (AMPs) during T. muris and Nippostrongylus brasiliensis infection are associated with the induction of Th2 responses in resistant mice. ${ }^{62,63}$ During T. muris infection, changes in expression of the AMP Ang4, as well as altered mucin expression patterns depend on signaling through IL-4Ra. ${ }^{64,65}$ The initiation of a type 2 response, even in the absence of live helminth infection, can be sufficient to modulate intestinal microbiota populations: administering mice IL-25, an initiating factor for type 2 immunity, ${ }^{66}$ can alter AMP expression levels and reduce the abundance of SFB, which replicates phenotypes seen in $N$. brasiliensis-infected mice. ${ }^{48}$ The effects of a type 2 response on AMP expression and mucus likely reflect the need for the host to stimulate tissue repair pathways as a result of damage caused during helminth infection. ${ }^{67}$ This is exemplified in the case of idiopathic chronic diarrhea in captive monkeys where Trichuris trichuria-induced IL4 was associated with repair of helminth-induced epithelial damage, and was accompanied with altered mucosal attachment of bacteria and microbiota composition. ${ }^{68}$ Additionally, helminth infection has been reported to cause shifts in microbiota populations independently of IL-4 and IL-13 signaling, as microbiota taxonomical changes have been reported to occur in IL-4Ra-deficient Heligmosomoides polygyrus-infected mice. $^{46}$

\section{METABOLIC CONSEQUENCES OF HELMINTH INFECTION}

Helminth infection has been widely reported to modify the metabolic capacity of their mammalian hosts (Table 1). Helminthelicited metabolic shifts are likely a combined result of altered host, bacterial, and helminth production of metabolites, as well as altered patterns of metabolite utilization, excretion, and absorption during helminth infection. Type 2 immune responses, such as those induced during helminth infection, ${ }^{23}$ can alter host metabolic function, ${ }^{69}$ as well as modifying bacterial microbiota populations, as discussed above.

The bacterial microbiota are major producers and utilizers of intestinal metabolites. ${ }^{40}$ Microbiota-derived metabolites have been shown to influence immune system development systemically and at a tissue level, and can fine-tune the immune response after the system has matured. ${ }^{70}$ While mammals lack the enzymes required to break down dietary fibers such as cellulose, certain microbiota species in the intestine can digest these carbohydrates through anaerobic fermentation. As a result, short-chain fatty acids (SCFAs) are released, a group of abundant metabolites (including acetate, butyrate, and propionate) that have been shown to influence multiple facets of immunity. Helminths themselves also both use and produce metabolites, ${ }^{11}$ and several helminth species are able to produce acetate. ${ }^{19,72}$

SCFAs can promote a regulatory immune response through stimulating the differentiation and suppressive capacity of Foxp $3^{+}$ Tregs in the intestine. ${ }^{73-75}$ Further, SCFAs can contribute to intestinal barrier function through stimulating mucus production, and can inhibit the release of inflammatory cytokines through the inhibition of NF-KB. ${ }^{76}$ In line with these effects on host immunity, a diet high in dietary fibers has been shown to dampen allergic airway inflammation and enhance tolerance to food antigens in mice. ${ }^{77-79}$ SCFA levels can influence susceptibility to bacterial infection, as microbial acetate production has been shown to protect against $E$. coli infection, likely through promoting barrier function. ${ }^{80}$

Helminth infection has repeatedly been reported to affect host carbohydrate metabolism (Table 1), in studies which either described predicted effects based on the detection of specific microbiota species, ${ }^{44,60,81-83}$ or in studies that directly measured end-products of carbohydrate metabolism. ${ }^{19,47,60,84-86}$ While carbohydrate metabolism and concentrations of SCFAs have been reported to be elevated during infections of mice with $H$. polygyrus $^{19}$ and goats with Haemonchus contortus, ${ }^{83}$ a reduced activity of these metabolic pathways has been reported during Ascaris infection in humans ${ }^{82}$ and T. suis infection in pigs. ${ }^{60}$ The differential helminth-elicited effects on carbohydrate metabolism may reflect different immune evasion strategies between helminth species. For example, dietary fermentable carbohydrates resulted in elevated cecal SCFA levels and accelerated the expulsion of $T$. suis in pigs, ${ }^{87}$ therefore Trichuris species may have evolved strategies to downregulate carbohydrate metabolism. In contrast, other helminth species might benefit from SCFA production through the induction of a regulatory immune response, ${ }^{73-75}$ which helps to prevent their own expulsion. The identification of key microbial metabolites that are affected during helminth infection (and characterization of their effect on immune responses) will likely provide a greater insight into the mechanisms by which helminth colonization affects immunity than associations between helminth infection and the presence of (potentially redundant) specific microbial taxa.

\section{A HELMINTH-MODIFIED MICROBIOME DIRECTLY AFFECTS HOST IMMUNITY}

The regulatory immune response that is established during helminth infection has the potential to ameliorate allergic disease, ${ }^{19,21,75,77,78}$ while a helminth-elicited tolerogenic environment could render the host more susceptible to microbial coinfections. ${ }^{7,9,13,14,88-90}$ Since intestinal helminths and the bacterial microbiota have a shared agenda of avoiding their own expulsion from the mammalian intestinal tract and have therefore each evolved mechanisms to modulate host immunity, several recent studies have examined the extent to which the immunomodulatory effects of helminth infection can be attributed to changes in the intestinal microbiome (Fig. 2). Transfer of a helminth-modified microbiota alone, in the absence of live helminth infection, has been shown to reduce airway inflammation in recipient mice in a mouse model of allergic asthma, suggesting that one of the mechanisms by which helminth can mediate allergic inflammation is through modifying the microbiome. ${ }^{19}$ Further, while infection with $H$. polygyrus reduced the severity of allergic lung inflammation in mice, protection was lost when $H$. polygyrus-infected mice were treated with antibiotics, indicating that an intact microbiota was required for protection from airway inflammation in this experimental system. ${ }^{19} \mathrm{~A}$ helminth-modified microbiota may also play a role in protecting from respiratory syncytial virus (RSV) infection: a pathogen which has been associated with an increased risk for allergic asthma development. ${ }^{91}$ In a mouse model, coinfection with $H$. polygyrus ameliorated RSV-induced lung pathology and reduced viral loads, and this was dependent on the presence of the microbiota, as helminth-mediated protection from RSV was lost in germ-free mice. ${ }^{10}$

In the intestinal tract, the bacterial microbiota can provide colonization resistance, therefore a loss of beneficial microbiota members has the potential to contribute to infectious disease. ${ }^{39}$ Since helminths alter the microbiota composition, ${ }^{41}$ helminth colonization may protect against bacterial-driven intestinal inflammation by promoting the expansion of protective members of the microbiota that inhibit outgrowth of inflammatory bacteria. For example, a helminth-induced bloom of Clostridiales species 


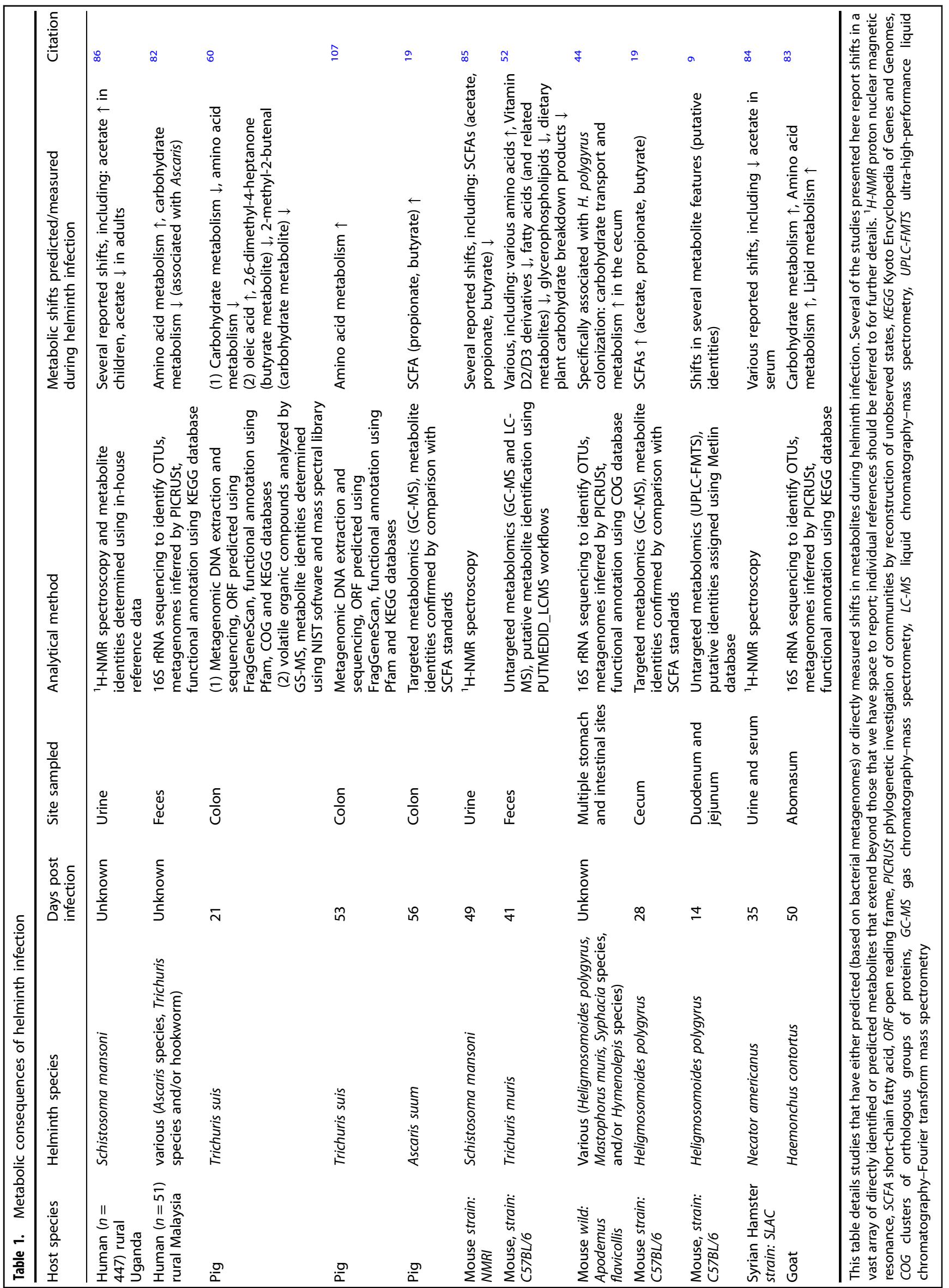




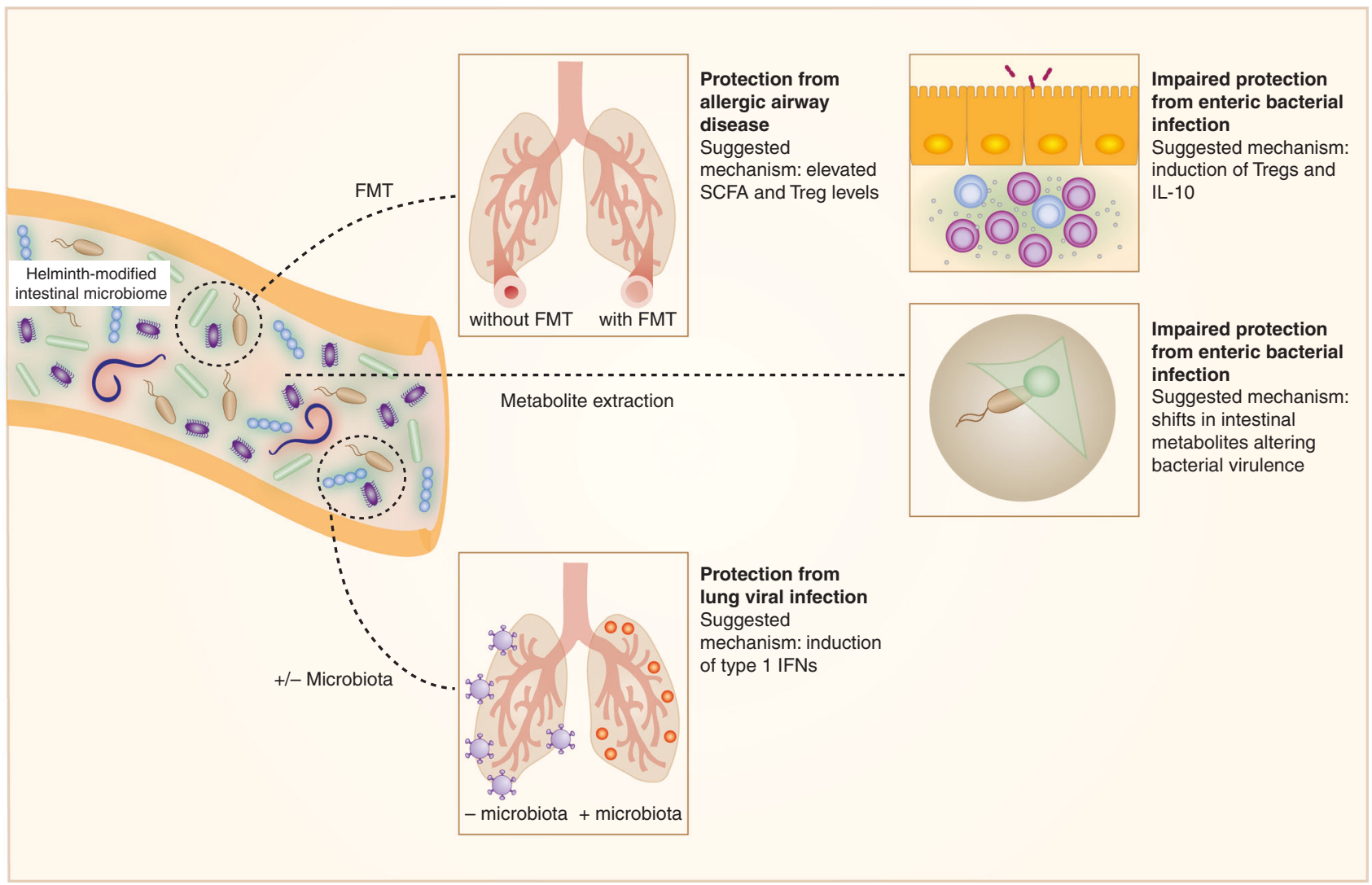

Fig. 2 A helminth-modified microbiome directly affects host immunity. The activity of a helminth-modified microbiome has been recently demonstrated in various mouse model systems. Transferring the fecal microbiota (FMT) from helminth-infected mice to non-helminth infected recipient mice can reduce levels of induced allergic airway inflammation in recipient mice, through the transfer of bacteria enriched in the production of short-chain fatty acids which likely contribute to Treg induction. ${ }^{19} \mathrm{~A}$ helminth-modified microbiome has been associated with impaired immunity to enteric bacterial infection, as FMT from helminth-infected mice resulted in elevated Treg frequencies and IL-10 levels in the gut-associated lymphoid tissue of recipient mice, alongside exacerbated Citrobacter-induced inflammation. ${ }^{90}$ In comparative studies of metabolites extracted from the small intestines of naïve or helminth infected mice, helminth-modified intestinal metabolites promoted the virulence of enteric infectious bacteria compared with metabolites from naïve mice. ${ }^{9}$ Finally, helminth infection can promote immunity to respiratory viral infection in mice through the induction of lung type I interferon, which was dependent on the helminthmodified microbiome, as protection from respiratory viral infection was lost in helminth-infected germ-free mice. ${ }^{10}$ FMT fecal microbiota transfer, SCFA short-chain fatty acids, Treg T regulatory cell, IFN interferon

blocked a bloom of pro-inflammatory Bacteroides vulgatus and ameliorated disease in a mouse model of IBD. ${ }^{61}$ However, the ability of a helminth-modified microbiota to protect from intestinal inflammation likely depends on the bacterial species that are driving inflammation. Supporting this, during a mouse model of Citrobacter rodentium-driven colitis, a helminth-modified microbiome exacerbated, rather than protected from, intestinal inflammation. ${ }^{90}$ Transfer of a $H$. polygyrus-modified microbiota in the absence of live helminth infection was sufficient to enhance colitis pathology initiated by $C$. rodentium infection. ${ }^{90}$ Together, these findings indicate that the helminth-modified microbiota can drive immunomodulation independently of live helminth infection in several scenarios, establishing an additional route by which helminth colonization causes host immunomodulation. The outcomes on disease development after exposure to a helminthmodified microbiota are heavily context-dependent.

\section{MECHANISMS BY WHICH A HELMINTH-MODIFIED MICROBIOME AFFECTS HOST IMMUNITY}

Direct effects of intestinal metabolites on pathogens

Helminths alter the composition and abundance of metabolites in the intestine (Table 1), which may directly affect the growth and/ or virulence of enteric pathogenic bacteria. ${ }^{92}$ While metabolites extracted from the intestine of naïve mice have been shown to inhibit the invasive capacity of Salmonella enterica serovar Typhimurium, ${ }^{93}$ metabolites from $H$. polygyrus-infected mice were unable to suppress $S$. Typhimurium invasion of epithelial cells. A loss of metabolite-mediated inhibition of bacterial invasion may contribute to the increased intestinal colonization of S. Typhimurium seen during helminth infection. ${ }^{9}$

Induction of regulatory immune responses by microbiotaproduced metabolites

Helminth suppression of allergic airway inflammation can be mediated, at least in part, by a helminth-modified microbiota that is enriched in the capacity for SCFA production. ${ }^{19}$ Evidencing this, $H$. polygyrus-infected mice showed a bloom in SCFA-fermenting Clostridial species and a corresponding increase in cecal SCFA levels, and transfer of a $H$. polygyrus-modified microbiota resulted in elevated SCFA levels in recipient mice. ${ }^{19}$ Antibiotic treatment of $H$. polygyrus-infected mice entirely abolished SCFA production and reverted the suppressive effects of helminth infection on airway inflammation. ${ }^{19}$ Further, the effects of helminth infection on allergic inflammation were dependent on expression of GPR41, a major SCFA receptor. ${ }^{19,77}$ A helminth-modified microbiota with a high capacity for SCFA production likely contributes to the amelioration of allergic airway inflammation through the induction of Tregs. ${ }^{21,75,78}$ In support of this, helminth-infected mice exhibited GPR41-dependent increases in IL-10 and TGF- $\beta$ levels in 
the lung during allergic airway inflammation. ${ }^{19}$ Microbial fermentation of dietary fibers may also modulate immunity during intestinal inflammation, since Treg expression of an additional SCFA receptor, GPR43, provided protection against a $T$ cell transfer-induced mouse model of colitis. ${ }^{74}$ In accordance with this, transfer of a helminth-modified microbiome boosted Treg frequencies and IL-10 expression in the mesenteric lymph nodes. $^{90}$ Tregs can contribute to effective immunity against $C$. rodentium through promoting Th17 induction, ${ }^{94}$ but in the context of a helminth-modified microbiota transfer, induction of Tregs has been associated with susceptibility to $C$. rodentium infection. ${ }^{90}$ Antibodies against CD25, and IL-10 neutralization, blocked the effects of a helminth-modified microbiota transfer on $C$. rodentium infection. ${ }^{90}$ It will be important for future studies to characterize further potential mechanisms by which a helminth-modified microbiota can promote host regulatory responses.

Microbiota-dependent stimulation of innate responses

Helminth colonization can also induce changes in the innate immune pathways that affect host resistance to microbial pathogens. Microbiota-dependent shifts during $H$. polygyrus infection induced type I interferon production in the lung which was required for protection from RSV infection. ${ }^{10}$ Protection from RSV was lost in RAG $-/-$ and IL-4Ra-/- mice, and in mice raised in germ-free conditions. ${ }^{10}$ Interestingly, protection against RSV infection in the lungs occurred after infection with irradiated $H$. polygyrus larvae, which were unable to develop into adult worms. ${ }^{10}$ Since $H$. polygyrus larval stages were sufficient to protect against viral infection, the authors proposed that the initial intestinal damage caused by larvae migrating into intestinal tissue and coincidental translocation of microbiota species into the tissue might evoke a systemic innate immune response, facilitating an antiviral response in the lungs. ${ }^{10}$

\section{CONCLUSION AND OUTLOOK}

Multiple mechanisms contribute to the modulation of host immunity during helminth infection. These include the products produced directly by helminths that have direct effects on host immune cells, ${ }^{27}$ as well as the indirect effects on host immunity, through an altered composition and function of the intestinal microbiome during helminth infection. The pathways by which a helminth-modified microbiome affect host immunity have only recently begun to be explored, and it will be important to evaluate the extent to which a helminth-modified microbiome contributes to long-term host immunomodulation following clearance of live helminth infection.

The fact that antimicrobial molecules have been described within the ES products of helminths ${ }^{55-57}$ suggests that helminths have an incentive to control the microbial species with which they share a niche. This may have evolved to protect the host from microbial exposure during helminth infection, or as a helminthdriven strategy for modulating host immune responses. ${ }^{95}$ While some aspects of anti-helminth host immunity are dependent on the microbiota, ${ }^{96}$ it is also possible that helminths have evolved the ability to expand those microbiota species that benefit their own survival. The hatching of $T$. muris eggs in the ceca of mice is dependent on the presence of microbiota, ${ }^{97}$ and supplementation with Lactobacilli species renders mice more susceptible to both $T$. muris and $H$. polygyrus infection. ${ }^{43,51}$ In addition, certain microbiota species could benefit from helminth infection, for example the abundance of Lactobacilli species is increased during chronic helminth infection. ${ }^{43}$ This indicates the existence of a mutualistic relationship between helminths and certain microbiota species, which could be a result of a shared aim: regulating mucosal immune responses in order to persist in the intestinal environment.
Helminths, microbiota species and mammals share a long history of co-evolution, during which a complex web of intercommunication has evolved. When attempting to understand the mechanistic pathways by which helminths manipulate host disease states, it will be vital to consider the multi-directional relationships between the host, helminths, and the microbiota. These interconnected pathways have been starting to be explored in human studies, ${ }^{98,99}$ however the challenge remains to discern causal relationships, for which animal models are proving to be invaluable. Examining the effects of a helminth-modified microbiome paves the way for therapeutic treatment options using preor probiotics, or microbiome-derived products, which could be used alone or in combination with helminth-derived products for the prevention or treatment of allergic, infectious, or inflammatory diseases.

\section{ACKNOWLEDGEMENTS}

Work in the authors' laboratory is supported by funds from the Natural Sciences and Engineering Research Council of Canada (NSERC) and the University of Victoria.

\section{AUTHOR CONTRIBUTIONS}

T.P.B. and L.A.R. both discussed the article content, wrote and edited drafts of the article, and approved the final contents of the article.

\section{ADDITIONAL INFORMATION}

Conflict of interest: The authors declare no competing financial interests.

Publisher's note: Springer Nature remains neutral with regard to jurisdictional claims in published maps and institutional affiliations.

\section{REFERENCES}

1. Varyani, F., Fleming, J. O. \& Maizels, R. M. Helminths in the gastrointestinal tract as modulators of immunity and pathology. Am. J. Physiol. Gastrointest. Liver Physiol. 312, G537-G549 (2017).

2. Harris, J. B. et al. Immunologic responses to Vibrio cholerae in patients coinfected with intestinal parasites in Bangladesh. PLoS Negl. Trop. Dis. 3, e403 (2009).

3. Elias, D., Mengistu, G., Akuffo, H. \& Britton, S. Are intestinal helminths risk factors for developing active tuberculosis? Trop. Med. Int. Heal. 11, 551-558 (2006).

4. Degarege, A., Legesse, M., Medhin, G., Animut, A. \& Erko, B. Malaria and related outcomes in patients with intestinal helminths: a cross-sectional study. BMC. Infect. Dis. 12, 291 (2012).

5. Resende, Co,T., Hirsch, C. S., Toossi, Z., Dietze, R. \& Ribeiro-Rodrigues, R. Intestinal helminth co-infection has a negative impact on both anti-Mycobacterium tuberculosis immunity and clinical response to tuberculosis therapy. Clin. Exp. Immunol. 147, 45-52 (2007).

6. Downs, J. A. et al. Urogenital schistosomiasis in women of reproductive age in Tanzania's Lake Victoria region. Am. J. Trop. Med. Hyg. 84, 364-369 (2011).

7. Osborne, L. C. et al. Virus-helminth coinfection reveals a microbiotaindependent mechanism of immunomodulation. Science 345, 578-582 (2014).

8. Marple, A. et al. Cutting edge: helminth coinfection blocks effector differentiation of CD8 T cells through alternate host Th2- and IL-10-mediated responses. J. Immunol. 198, 634-639 (2016).

9. Reynolds, L. A. et al. Enteric helminths promote Salmonella co-infection by altering the intestinal metabolome. J. Infect. Dis. 215, 1245-1254 (2017).

10. McFarlane, A. J. et al. Enteric helminth-induced type I interferon signaling protects against pulmonary virus infection through interaction with the microbiota. J. Allergy Clin. Immunol. 140, 1068-1078 (2017).

11. Legesse, M., Erko, B. \& Balcha, F. Increased parasitaemia and delayed parasite clearance in Schistosoma mansoni and Plasmodium berghei co-infected mice. Acta Trop. 91, 161-166 (2004)

12. Chen, $\mathrm{C}$. et al. Concurrent infection with an intestinal helminth parasite impairs host resistance to enteric Citrobacter rodentium and enhances Citrobacterinduced colitis in mice. Infect. Immun. 73, 5468-5481 (2005).

13. Reese, T. A. et al. Helminth infection reactivates latent gamma-herpesvirus via cytokine competition at a viral promoter. Science 345, 573-577 (2014). 
14. Hsieh, Y. J., Fu, C. L. \& Hsieh, M. H. Helminth-induced interleukin-4 abrogates invariant natural killer $\mathrm{T}$ cell activation-associated clearance of bacterial infection. Infect. Immun. 82, 2087-2097 (2014).

15. Su, L. et al. Coinfection with an intestinal helminth impairs host innate immunity against Salmonella enterica serovar typhimurium and exacerbates intestinal inflammation in mice. Infect. Immun. 82, 3855-3866 (2014).

16. Salgame, P., Yap, G. S. \& Gause, W. C. Effect of helminth-induced immunity on infections with microbial pathogens. Nat. Immunol. 14, 1118-1126 (2013).

17. Yazdanbakhsh, M., Van Den Biggelaar, A. \& Maizels, R. M. Th2 responses without atopy: immunoregulation in chronic helminth infections and reduced allergic disease. Trends Immunol. 22, 372-377 (2001)

18. Trujillo-Vargas, C. M. et al. Helminth-derived products inhibit the development of allergic responses in mice. Am. J. Respir. Crit. Care. Med. 175, 336-344 (2007).

19. Zaiss, M. M. et al. The intestinal microbiota contributes to the ability of helminths to modulate allergic inflammation. Immunity 43, 998-1010 (2015).

20. McSorley, H. J. et al. Suppression of type 2 immunity and allergic airway inflammation by secreted products of the helminth Heligmosomoides polygyrus. Eur. J. Immunol. 42, 2667-2682 (2012).

21. Wilson, M. S. et al. Suppression of allergic airway inflammation by helminthinduced regulatory T cells. J. Exp. Med. 202, 1199-1212 (2005).

22. Lambrecht, B. N. \& Hammad, H. The immunology of the allergy epidemic and the hygiene hypothesis. Nat. Immunol. 18, 1076-1083 (2017).

23. Grencis, R. K. Immunity to helminths: resistance, regulation, and susceptibility to gastrointestinal nematodes. Annu. Rev. Immunol. 33, 201-225 (2015).

24. McSorley, H. J., Hewitson, J. P. \& Maizels, R. M. Immunomodulation by helminth parasites: defining mechanisms and mediators. Int. J. Parasitol. 43, 301-310 (2013).

25. Buck, A. H. et al. Exosomes secreted by nematode parasites transfer small RNAs to mammalian cells and modulate innate immunity. Nat. Commun. 5, 5488 (2014).

26. Johnston, C. J. C. et al. A structurally distinct TGF- $\beta$ mimic from an intestinal helminth parasite potently induces regulatory T cells. Nat. Commun. 8, 1741 (2017).

27. Maizels, R. M. \& McSorley, H. J. Regulation of the host immune system by helminth parasites. J. Allergy Clin. Immunol. 138, 666-675 (2016).

28. O'Hara, A. M. \& Shanahan, F. The gut flora as a forgotten organ. EMBO. Rep. 7, 688-693 (2006).

29. Hooper, L. V., Littman, D. R. \& Macpherson, A. J. Interactions between the microbiota and the immune system. Science 336, 1268-1273 (2012).

30. Ivanov, I. I. et al. Specific microbiota direct the differentiation of IL-17-producing T-helper cells in the mucosa of the small intestine. Cell Host. Microbe 4, 337-349 (2008).

31. Ivanov, I. I. et al. Induction of intestinal Th17 cells by segmented filamentous bacteria. Cell 139, 485-498 (2009).

32. Gaboriau-Routhiau, V. et al. The key role of segmented filamentous bacteria in the coordinated maturation of gut helper $\mathrm{T}$ cell responses. Immunity 31, 677-689 (2009).

33. Atarashi, K. et al. Induction of colonic regulatory T cells by indigenous Clostridium species. Science 331, 337-341 (2011).

34. Ohnmacht, C. et al. The microbiota regulates type 2 immunity through $\mathrm{ROR}^{\mathrm{V}} \mathrm{t}^{+}$ T cells. Science 349, 989-993 (2015).

35. Atarashi, K. et al. Treg induction by a rationally selected mixture of Clostridia strains from the human microbiota. Nature 500, 232-236 (2013).

36. Reynolds, L. A. \& Finlay, B. B. A case for antibiotic perturbation of the microbiota leading to allergy development. Expert Rev. Clin. Immunol. 9, 1019-1030 (2013).

37. Nell, S., Suerbaum, S. \& Josenhans, C. The impact of the microbiota on the pathogenesis of IBD: lessons from mouse infection models. Nat. Rev. Microbiol. 8, 564-577 (2010)

38. Ubeda, C., Djukovic, A. \& Isaac, S. Roles of the intestinal microbiota in pathogen protection. Clin. Transl. Immunol. 6, e128 (2017).

39. Kamada, N., Seo, S.-U., Chen, G. Y. \& Núñez, G. Role of the gut microbiota in immunity and inflammatory disease. Nat. Rev. Immunol. 13, 321-335 (2013).

40. Rowland, I. et al. Gut microbiota functions: metabolism of nutrients and other food components. Eur. J. Nutr. 0, 1-24 (2017)

41. Peachey, L. E., Jenkins, T. P. \& Cantacessi, C. This gut ain't big enough for both of us. Or is it? Helminth-microbiota interactions in veterinary species. Trends Parasitol. 33, 619-632 (2017).

42. Wells, J. M. Immunomodulatory mechanisms of lactobacilli. Microb. Cell Fact. 10 S17 (2011).

43. Reynolds, L. A. et al. Commensal-pathogen interactions in the intestinal tract lactobacilli promote infection with, and are promoted by, helminth parasites. Gut Microbes 5, 522-532 (2014).

44. Kreisinger, J., Bastien, G., Hauffe, H. C., Marchesi, J. \& Perkins, S. E. Interactions between multiple helminths and the gut microbiota in wild rodents. Philos. Trans. R. Soc. B Biol. Sci. 370, 20140295 (2015).
45. Walk, S. T., Blum, A. M., Ewing, S. A. S., Weinstock, J. V. \& Young, V. B. Alteration of the murine gut microbiota during infection with the parasitic helminth Heligmosomoides polygyrus. Inflamm. Bowel Dis. 16, 1841-1849 (2010).

46. Rausch, $\mathrm{S}$. et al. Small intestinal nematode infection of mice is associated with increased enterobacterial loads alongside the intestinal tract. PLOS ONE 8, 1-13 (2013).

47. Holm, J. B. et al. Chronic Trichuris muris infection decreases diversity of the intestinal microbiota and concomitantly increases the abundance of lactobacilli. PLOS ONE 10, 1-22 (2015).

48. Fricke, W. F. et al. Type 2 immunity-dependent reduction of segmented filamentous bacteria in mice infected with the helminthic parasite Nippostrongylus brasiliensis. Microbiome 3, 40 (2015).

49. Plieskatt, J. L. et al. Infection with the carcinogenic liver fluke Opisthorchis viverrini modifies intestinal and biliary microbiome. FASEB J. 27, 4572-4584 (2013).

50. Duarte, A. M. et al. Helminth infections and gut microbiota - a feline perspective. Parasit. Vectors 9, 625 (2016).

51. Dea-Ayuela, M. A., Rama-Iñiguez, S. \& Bolás-Fernandez, F. Enhanced susceptibility to Trichuris muris infection of $\mathrm{B} 10 \mathrm{Br}$ mice treated with the probiotic Lactobacillus casei. Int. Immunopharmacol. 8, 28-35 (2008).

52. Houlden, A. et al. Chronic Trichuris muris infection in $\mathrm{C} 57 \mathrm{BL} / 6$ mice causes significant changes in host microbiota and metabolome: effects reversed by pathogen clearance. PLOS ONE 10, e0125945 (2015).

53. Conlon, M. A. \& Bird, A. R. The impact of diet and lifestyle on gut microbiota and human health. Nutrients 7, 17-44 (2014).

54. Harrison, C. A. \& Taren, D. How poverty affects diet to shape the microbiota and chronic disease. Nat. Rev. Immunol. (2017) https://doi.org/10.1038/nri.2017.121.

55. Kato, Y. \& Komatsu, S. ASABF, a novel cysteine-rich antibacterial peptide isolated from the nematode Ascaris suum: purification, primary structure, and molecular cloning of cDNA. J. Biol. Chem. 271, 30493-30498 (1996).

56. Hewitson, J. P. et al. Proteomic analysis of secretory products from the model gastrointestinal nematode Heligmosomoides polygyrus reveals dominance of Venom Allergen-Like (VAL) proteins. J. Proteom. 74, 1573-1594 (2011).

57. Abner, S. R., Parthasarathy, G., Hill, D. E. \& Mansfield, L. S. Trichuris suis: detection of antibacterial activity in excretory-secretory products from adults. Exp. Parasitol. 99, 26-36 (2001)

58. Shea-Donohue, T. et al. The role of IL- 4 in Heligmosomoides polygyrus-induced alterations in murine intestinal epithelial cell function. J. Immunol. 167, 2234-2239 (2001).

59. Robertson, B. R. et al. Mucispirillum schaedleri gen. nov., sp. nov., a spiral-shaped bacterium colonizing the mucus layer of the gastrointestinal tract of laboratory rodents. Int. J. Syst. Evol. Microbiol. 55, 1199-1204 (2005).

60. Li, R. W. et al. Alterations in the porcine colon microbiota induced by the gastrointestinal nematode Trichuris suis. Infect. Immun. 80, 2150-2157 (2012).

61. Ramanan, D. et al. Helminth infection promotes colonization resistance via type 2 immunity. Science 352, 608-612 (2016)

62. Datta, R. et al. Identification of novel genes in intestinal tissue that are regulated after infection with an intestinal nematode parasite. Infect. Immun. 73, 4025-4033 (2005).

63. D'Elia, R. et al. Expulsion of Trichuris muris is associated with increased expression of angiogenin 4 in the gut and increased acidity of mucins within the goblet cell. BMC Genom. 10, 492 (2009).

64. Forman, R. A. et al. The goblet cell is the cellular source of the anti-microbial angiogenin 4 in the large intestine post Trichuris muris infection. PLOS ONE 7, 4-10 (2012).

65. Hasnain, S. Z. et al. Muc5ac: a critical component mediating the rejection of enteric nematodes. J. Exp. Med. 208, 893-900 (2011).

66. Saenz, S. A., Taylor, B. C. \& Artis, D. Welcome to the neighborhood: epithelial cellderived cytokines license innate and adaptive immune responses at mucosal sites. Immunol. Rev. 226, 172-190 (2008).

67. Peterson, L. W. \& Artis, D. Intestinal epithelial cells: regulators of barrier function and immune homeostasis. Nat. Rev. Immunol. 14, 141-153 (2014).

68. Broadhurst, M. J. et al. Therapeutic helminth infection of macaques with idiopathic chronic diarrhea alters the inflammatory signature and mucosal microbiota of the colon. PLoS Pathog. 8, e1003000 (2012).

69. Brestoff, J. R. \& Artis, D. Immune regulation of metabolic homeostasis in health and disease. Cell 161, 146-160 (2015).

70. Levy, M., Thaiss, C. A. \& Elinav, E. Metabolites: messengers between the microbiota and the immune system. Genes Dev. 30, 1589-1597 (2016).

71. Guigas, B. \& Molofsky, A. B. A worm of one's own: how helminths modulate host adipose tissue function and metabolism. Trends Parasitol. 31, 435-441 (2015).

72. Tielens, A. G. M., van Grinsven, K. W. A., Henze, K., van Hellemond, J. J. \& Martin, W. Acetate formation in the energy metabolism of parasitic helminths and protists. Int. J. Parasitol. 40, 387-397 (2010). 
73. Furusawa, Y., Obata, Y. \& Hase, K. Commensal microbiota regulates T cell fate decision in the gut. Semin. Immunopathol. 37, 17-25 (2015).

74. Smith, P. M. et al. The microbial metabolites, short-chain fatty acids, regulate colonic Treg cell homeostasis. Science 341, 569-573 (2013).

75. Arpaia, N. et al. Metabolites produced by commensal bacteria promote peripheral regulatory T-cell generation. Nature 504, 451-455 (2013).

76. Thorburn, A. N., Macia, L. \& Mackay, C. R. Diet, metabolites, and 'Western-lifestyle' inflammatory diseases. Immunity 40, 833-842 (2014).

77. Trompette, A. et al. Gut microbiota metabolism of dietary fiber influences allergic airway disease and hematopoiesis. Nat. Med. 20, 159-166 (2014).

78. Thorburn, A. N. et al. Evidence that asthma is a developmental origin disease influenced by maternal diet and bacterial metabolites. Nat. Commun. 6, 7320 (2015).

79. Tan, J. et al. Dietary fiber and bacterial SCFA enhance oral tolerance and protect against food allergy through diverse cellular pathways. Cell Rep. 15, 2809-2824 (2016).

80. Fukuda, S. et al. Bifidobacteria can protect from enteropathogenic infection through production of acetate. Nature 469, 543-547 (2011).

81. Cattadori, I. M. et al. Impact of helminth infections and nutritional constraints on the small intestine microbiota. PLoS ONE 11, e0159770 (2016).

82. Lee, S. C. et al. Helminth colonization is associated with increased diversity of the gut microbiota. PLoS Negl. Trop. Dis. 8, e2880 (2014).

83. Li, R. W. et al. The effect of helminth infection on the microbial composition and structure of the caprine abomasal microbiome. Sci. Rep. 6, 20606 (2016).

84. Wang, Y. et al. Systems metabolic effects of a Necator americanus infection in Syrian hamster. J. Proteome Res. 8, 5442-5450 (2009).

85. Wang, Y. et al. Metabonomic investigations in mice infected with Schistosoma mansoni: an approach for biomarker identification. Proc. Natl Acad. Sci. USA 101, 12676-12681 (2004).

86. Balog, C. I. et al. Metabonomic investigation of human Schistosoma mansoni infection. Mol. Biosyst. 7, 1473-1480 (2011).

87. Thomsen, L. E., Petkevičius, S., Bach Knudsen, K. E. \& Roepstorff, A. The influence of dietary carbohydrates on experimental infection with Trichuris suis in pigs. Parasitology 131, 857-865 (2005)

88. Chen, C.-C., Louie, S., McCormick, B. A., Walker, W. A. \& Shi, H. N. Helminthprimed dendritic cells alter the host response to enteric bacterial infection. $J$. Immunol. 176, 472-483 (2006).

89. Weng, $M$. et al. Alternatively activated macrophages in intestinal helminth infection: effects on concurrent bacterial colitis. J. Immunol. 179, 4721-4731 (2007)

90. Su, C. et al. Helminth-induced alterations of the gut microbiota exacerbate bacterial colitis. Mucosal Immunol. 1-14 (2017). https://doi.org/10.1038/ mi. 2017.20

91. Wu, P. \& Hartert, T. Evidence for a causal relationship between respiratory syncytial virus infection and asthma. Natl. Inst. Heal. 9, 731-745 (2011).
92. Vogt, S. L., Peña-Díaz, J. \& Finlay, B. B. Chemical communication in the gut: effects of microbiota-generated metabolites on gastrointestinal bacterial pathogens. Anaerobe 34, 106-115 (2015).

93. Peixoto, R. J. M. et al. Repression of Salmonella host cell invasion by aromatic small molecules from the human fecal metabolome. Appl. Environ. Microbiol. 83, AEM.01148-17 (2017).

94. Wang, Z. et al. Regulatory $T$ cells promote a protective Th17-associated immune response to intestinal bacterial infection with C. rodentium. Mucosal Immunol. 7, 1290-1301 (2014).

95. Cotton, S., Donnelly, S., Robinson, M. W., Dalton, J. P. \& Thivierge, K. Defense peptides secreted by helminth pathogens: antimicrobial and/or immunomodulator molecules? Front. Immunol. 3, 1-7 (2012).

96. Entwistle, L. J. et al. Epithelial-cell-derived phospholipase A 2 group 1B is an endogenous anthelmintic. Cell Host Microbe 22, 484-493 (2017).

97. Hayes, K. S. et al. Exploitation of the intestinal microflora by the parasitic nematode trichuris muris. Science 328, 1391-1394 (2010).

98. Giacomin, P. et al. Changes in duodenal tissue-associated microbiota following hookworm infection and consecutive gluten challenges in humans with coeliac disease. Sci. Rep. 6, 1-10 (2016).

99. Giacomin, P. et al. Experimental hookworm infection and escalating gluten challenges are associated with increased microbial richness in celiac subjects. Sci. Rep. 5, 4-11 (2015).

100. Marillier, R. G. et al. IL-4/IL-13 independent goblet cell hyperplasia in experimental helminth infections. BMC Immunol. 9, 11 (2008).

101. Tsubokawa, D. et al. Induction of $\mathrm{Sd}^{\mathrm{a}}$-sialomucin and sulfated $\mathrm{H}$-sulfomucin in mouse small intestinal mucosa by infection with parasitic helminth. Exp. Parasitol. 153, 165-173 (2015).

102. Hasnain, S. Z., McGuckin, M. A., Grencis, R. K. \& Thornton, D. J. Serine protease(s) secreted by the nematode Trichuris muris degrade the mucus barrier. PLoS Negl. Trop. Dis. 6, e1856 (2012)

103. Venugopal, P. G., Nutman, T. B. \& Semnani, R. T. Activation and regulation of Toll-like receptors (TLRs) by helminth parasites. Immunol. Res. 43, 252-263 (2009).

104. Sun, S. et al. Toll-like receptor activation by helminths or helminth products to alleviate inflammatory bowel disease. Parasit. Vectors 4, 186 (2011).

105. Kosik-Bogacka, D. I. et al. Hymenolepis diminuta: analysis of the expression of Toll-like receptor genes (TLR2 and TLR4) in the small and large intestines of rats. Exp. Parasitol. 130, 261-266 (2012).

106. Zakeri, A., Borji, H. \& Haghparast, A. Interaction between helminths and toll-like receptors: possibilities and potentials for asthma therapy. Int. Rev. Immunol. 35, 219-248 (2016).

107. Wu, S. et al. Worm burden-dependent disruption of the porcine colon microbiota by trichuris suis infection. PLoS ONE 7, e35470 (2012). 